\title{
Theoretical Formulation for an Electrically Small Microstrip Patch Antenna Loaded with Negative Index Materials
}

\author{
J. S. Petko* and D. H. Werner \\ The Pennsylvania State University \\ Department of Electrical Engineering and \\ Applied Research Laboratory \\ jsp160@psu.edu and dhw@psu.edu
}

\section{Introduction}

There has been a considerable amount of recent interest in the class of composite metamaterials that possess simultaneously negative permeability and permittivity, causing them to exhibit a negative index of refraction [1,2]. This type of metamaterial is known as a Negative Index Material (NIM). An application of NIMs to increasing the power radiated from electrically small antennas has been suggested by Ziolkowski and Kipple [3]. Moreover, Engheta recently proposed in [4] the use of NIMs to design thin sub-wavelength cavity resonators. It was found that a miniature resonator could be created by filling the cavity with a region of NIM and a region of conventional Positive Index Material (PIM), leading to a "resonance condition" dependent upon the permeabilities of the two embedded materials. In this paper we expand on this principle by introducing a method to miniaturize a circular microstrip patch antenna that uses NIM loading. This is accomplished by including two distinct regions inside the cavity of a microstrip patch antenna, one consisting of a NIM and one consisting of a conventional PIM. By applying the boundary conditions commonly associated with the cavity model of a patch antenna, a "resonance condition" is found that is dependent only on the permittivities of the two embedded substrate materials.

\section{Formulation}

Our examination of the resonance condition for a miniature NIM loaded circular microstrip patch begins by considering the cavity model for the antenna. The cavity model replaces the patch geometry by an equivalent cavity using PEC material for the top and bottom boundary and PMC material for the sidewall of the cavity. This leads to the following boundary conditions for the fields:

$$
\begin{gathered}
E_{\rho}(0 \leq \rho \leq a, 0 \leq \varphi \leq 2 \pi, z=0, h)=0 \\
H_{\varphi}(\rho=a, 0 \leq \varphi \leq 2 \pi, 0 \leq z \leq h)=0
\end{gathered}
$$

In the case of the miniature microstrip patch antenna, the cavity is divided into two regions, one inner cylinder of NIM and one outer shell of conventional PIM. The geometry of this cavity model is illustrated in Figure 1 where $a$ is the radius of the microstrip patch, $b$ is the radius of the NIM cylinder, $\varepsilon_{1}$ and $\mu_{1}$ are the permittivity and permeability of the NIM, whereas $\varepsilon_{2}$ and $\mu_{2}$ are the permittivity and permeability of the PIM. Throughout the formulation the NIM is assumed to be contained in region 1 (denoted by a subscript or superscript 1) and the PIM is assumed to be contained in region 2 (denoted by a subscript or superscript 2 ). It is clear that only $\mathrm{TM}^{z}$ modes are supported by the cavity. Therefore, the vector potential may be expressed in the form $\mathbf{A}=A_{z} \hat{\mathbf{z}}$, which must satisfy the homogeneous wave equation:

$$
\begin{aligned}
& \nabla^{2} A_{z}^{1}+k_{1}^{2} A_{z}^{1}=0 \text { in region } 1 \\
& \nabla^{2} A_{z}^{2}+k_{2}^{2} A_{z}^{2}=0 \text { in region } 2
\end{aligned}
$$

where $k_{1}$ and $k_{2}$ are defined by $k_{n}{ }^{2}=k_{\rho n}{ }^{2}+k_{z n}{ }^{2}$ while $A_{z}^{1}$ and $A_{z}^{2}$ are in the general form of: 


$$
A_{z}^{n}\left(k_{\rho n}, m, k_{z n} ; \rho, \varphi, z\right)=B_{m}\left(k_{\rho n} \rho\right) h(m \varphi) h\left(k_{z n} z\right)
$$

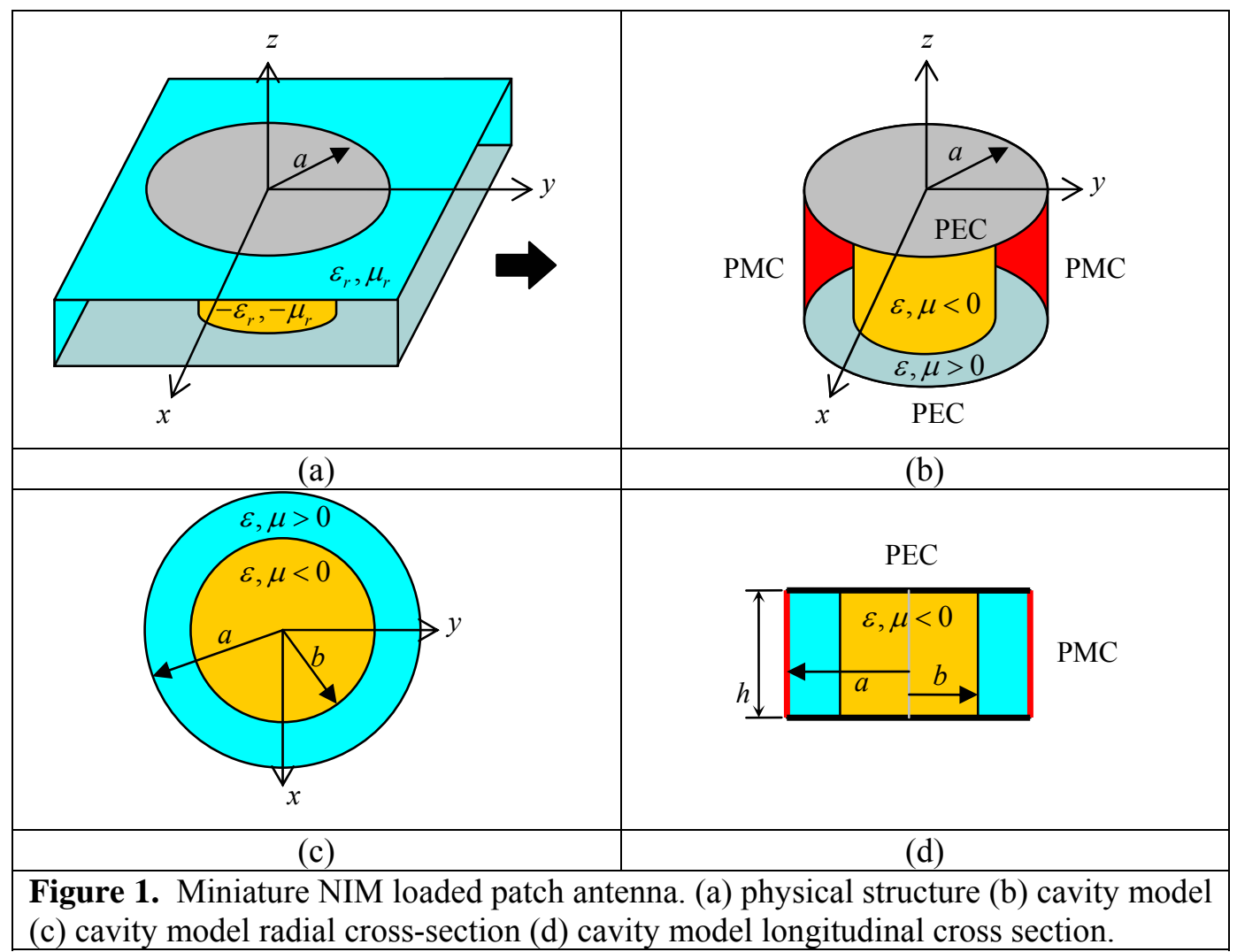

Note that when applying boundary condition (1a) to the radial electric field resulting from either (2a) or (2b), $h\left(k_{z n} z\right)$ must be in the form of $\cos \left(k_{z n} z\right)$ where $\left|k_{z n}\right|=p \pi / h$. Also, in this case the terms $B_{m}\left(k_{\rho 1} \rho\right)$ are represented by the Bessel functions of the first kind of order $m$ given by $J_{m}\left(k_{\rho 1} \rho\right)$. In addition to the boundary conditions existing at the walls of the cavity, there are conditions associated with the boundary between the NIM (region 1) and PIM (region 2):

$$
\begin{gathered}
E_{z}^{1}(\rho=b, 0 \leq \varphi \leq 2 \pi, 0 \leq z \leq h)=E_{z}^{2}(\rho=b, 0 \leq \varphi \leq 2 \pi, 0 \leq z \leq h) \\
H_{\varphi}^{1}(\rho=b, 0 \leq \varphi \leq 2 \pi, 0 \leq z \leq h)=H_{\varphi}^{2}(\rho=b, 0 \leq \varphi \leq 2 \pi, 0 \leq z \leq h) \\
k_{\rho 2} b+\theta_{b}=k_{\rho 1} b
\end{gathered}
$$

where the term $\theta_{b}$ is used to guarantee constant phase across the boundary.

At this point, the axial magnetic field equations resulting from (2a) and (2b) are inserted into boundary conditions (4b) along with (5) to yield

$$
\begin{aligned}
& -\frac{k_{\rho 1}}{\mu_{1}} A_{m n p}^{1} J_{m}^{\prime}\left(k_{\rho 1} b\right)\left[C_{1} \cos (m \varphi)+D_{1} \sin (m \varphi)\right] \cos \left(k_{z 1} z\right)= \\
& -\frac{k_{\rho 2}}{\mu_{2}}\left[A_{m n p}^{2} J_{m}^{\prime}\left(k_{\rho 2} b+\theta_{b}\right)+B_{m n}^{2} Y_{m}^{\prime}\left(k_{\rho 2} b+\theta_{b}\right)\right]\left[C_{2} \cos (m \varphi)+D_{2} \sin (m \varphi)\right] \cos \left(k_{z 2} z\right)
\end{aligned}
$$

This equation is valid given that, $C_{1}=C_{2} \equiv C, D_{I}=D_{2} \equiv D$, and $\left|k_{z 1}\right|=\left|k_{z 2}\right| \equiv\left|k_{z}\right|$. From this point the special case for the lowest-order mode when $m=0$ and $k_{z}=0$ will be examined, which simplifies the vector potential expressions to the form: 


$$
\begin{gathered}
A_{z}^{1}\left(k_{1}, 0,0 ; \rho, \varphi, z\right)=A_{0 n 0}^{1} J_{0}\left(k_{1} \rho\right) \\
A_{z}^{2}\left(k_{2}, 0,0 ; \rho, \varphi, z\right)=\left[A_{0 n 0}^{2} J_{0}\left(k_{2} \rho+\theta_{b}\right)+B_{0 n 0}^{2} Y_{0}\left(k_{2} \rho+\theta_{b}\right)\right]
\end{gathered}
$$

Since the wavenumber of the NIM is negative, the argument of the Bessel function is less than zero at the boundary $b$. Also, the argument of the Bessel functions should be the same on each side of the boundary. Therefore it is possible to have a case where the Bessel function arguments at the edge of the cavity are equal to zero. Thus we define the zero order resonance condition as

$$
k_{\rho 2} a+\theta_{b}=0
$$

Application of the boundary condition (1b) to (7b), leads to the conclusion that $B_{0 n 0}^{2}=0$. Hence, under these conditions, the fields must be equal to:

$$
\begin{array}{lll}
E_{z}^{1}=-j \omega E_{o}^{1} J_{0}\left(-n_{1} k_{o} \rho\right) & \text { (9a) } & E_{z}^{2}=-j \omega E_{o}^{2} J_{0}\left(n_{2} k_{0} \rho-n_{2} k_{o} a\right) \\
H_{\varphi}^{1}=-\frac{n_{1} k_{o}}{\mu_{1}} E_{o}^{1} J_{1}\left(-n_{1} k_{o} \rho\right) & \text { (9c) } & H_{\varphi}^{2}=-\frac{n_{2} k_{o}}{\mu_{2}} E_{o}^{2} J_{1}\left(n_{2} k_{o} \rho-n_{2} k_{o} a\right)
\end{array}
$$

where $k_{1}=\omega \sqrt{\mu_{1} \varepsilon_{1}}=-n_{1} k_{o}, k_{2}=\omega \sqrt{\mu_{2} \varepsilon_{2}}=n_{2} k_{o}$, and $k_{o}$ is the free space wavenumber. Applying boundary conditions (4) to (9) yields the following set of equations:

$$
\begin{gathered}
E_{o}^{1} J_{0}\left(n_{1} k_{o} b\right)-E_{o}^{2} J_{0}\left(n_{2} k_{o}(a-b)\right)=0 \\
\frac{n_{1}}{\mu_{1}} E_{o}^{1} J_{1}\left(n_{1} k_{o} b\right)+\frac{n_{2}}{\mu_{2}} E_{o}^{2} J_{1}\left(n_{2} k_{o}(a-b)\right)=0
\end{gathered}
$$

In order for these relationships to be true, the determinant must be equal to zero such that

$$
\frac{n_{2}}{\mu_{2}} J_{0}\left(n_{1} k_{o} b\right) J_{1}\left(n_{2} k_{o}(a-b)\right)+\frac{n_{1}}{\mu_{1}} J_{0}\left(n_{2} k_{o}(a-b)\right) J_{1}\left(n_{1} k_{o} b\right)=0
$$

This leads to the condition

$$
\frac{J_{0}\left(n_{1} k_{o} b\right) J_{1}\left(n_{2} k_{o}(a-b)\right)}{J_{1}\left(n_{1} k_{o} b\right) J_{0}\left(n_{2} k_{o}(a-b)\right)}=\frac{n_{1}}{n_{2}} \frac{\left|\mu_{2}\right|}{\left|\mu_{1}\right|}
$$

Finally, by applying the small argument approximation,

$$
J_{n}(z) \approx\left(\frac{z}{2}\right)^{n} \frac{1}{n !}
$$

equation (12) can be reduced to a useful form given by:

$$
\frac{(a-b)}{b} \approx \frac{n_{1}^{2}}{n_{2}^{2}} \frac{\left|\mu_{2}\right|}{\left|\mu_{1}\right|}=\frac{\left|\varepsilon_{1}\right|}{\left|\varepsilon_{2}\right|}
$$

As long as this relationship holds, where $\varepsilon_{1}$ and $\mu_{1}$ are both less than zero, then the zero order resonance condition exists.

\section{Discussion}

The zero order resonance condition is independent of frequency if the size of the patch is electrically small. In addition, from equation (12), the zero order resonance condition exists if $b=1 / 2 a, \varepsilon_{1}=-\varepsilon_{2}$, and $\mu_{1}=-\mu_{2}$, regardless of whether the antenna is electrically small or not. In this case, the bandwidth of the patch antenna is theoretically infinite for nondispersive materials. To observe this theoretical infinite bandwidth condition, we consider in Figure 2 the operation of a patch with the properties $b=1 / 2 a, \varepsilon_{1}=-\varepsilon_{2}$, and $\mu_{1}=-$ $\mu_{2}$. The boundary between the two regions of the patch antenna is clearly shown as a circle in the contour plots of the fields. Plots (a) and (c) illustrate the values of $E_{z}$ for cavities having $k=0.2 / \lambda$ and $k=4.0 / \lambda$ respectively. One can see that the electric fields are 
the same on each side of the NIM/PIM boundary and greatest in value at the center and outside edge of the cavity. Plots (b) and (d) show vector plots of $H_{\varphi}$ for cavities having $k=0.2 / \lambda$ and $k=4.0 / \lambda$ respectively. One can see that the magnetic fields are the same on each side of the NIM/PIM boundary and the fields at the outside edge of the patch are zero, agreeing with the PMC boundary condition (1b). In practice, NIM materials are typically dispersive and will therefore have a finite bandwidth. In such cases one can use equation (14) to create electrically small narrowband designs based solely on the permittivities of available materials (both PIM and NIM).

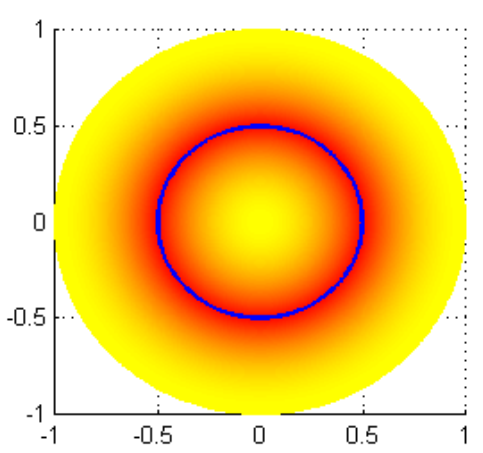

(a)

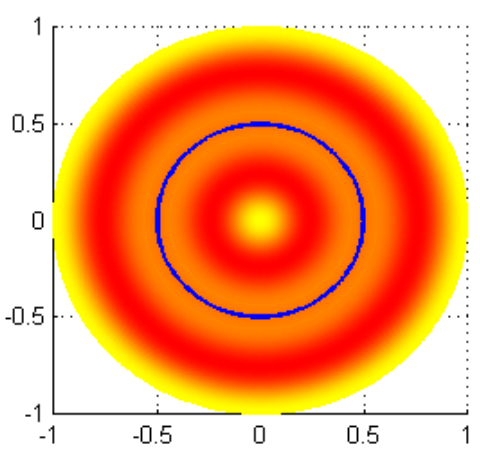

(c)
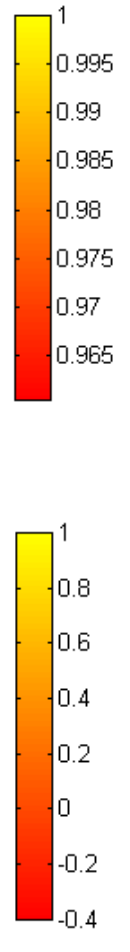

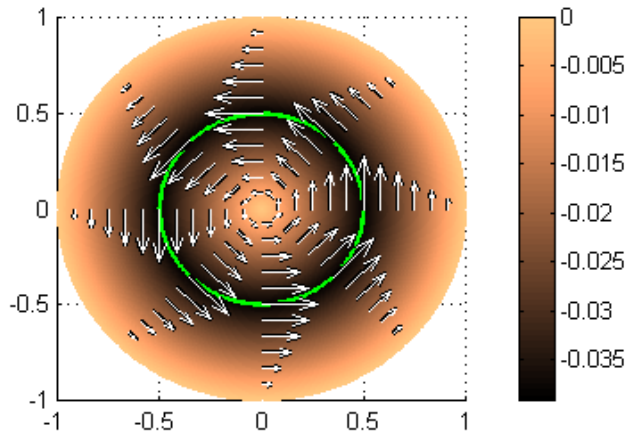

(b)

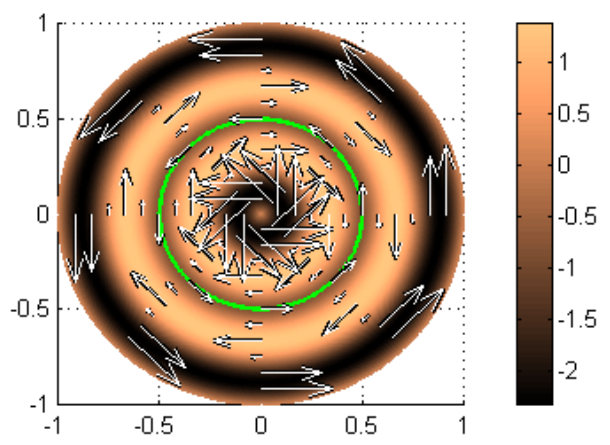

(d)

Figure 2. Two resonances for a NIM patch with $b=1 / 2 \quad a, \varepsilon_{1}=-\varepsilon_{2}, \mu_{1}=-\mu_{2}$, using the cavity model. The electric (a) and magnetic (b) fields for a resonance with $k=0.2 / \lambda$ are shown in the first set of figures, while the electric (c) and magnetic (d) fields for a resonance with $k=4 / \lambda$ are shown in the second set of figures.

\section{References}

[1] V. G. Vesalago, "The Electrodynamics of Substances with Simultaneously Negative Values of $\varepsilon$ and $\mu$,"Sov. Phys. USPEKHI, pp. 509-514, 1968.

[2] D. R. Smith, W. J. Padilla, D. C. Vier, S. C. Nemat-Nasser, and S. Schultz, "Composite Medium with Simultaneously Negative Permeability and Permittivity," Phys. Rev. Lett., Vol. 84, No. 10, pp. 4184-4187, 2000.

[3] R. W. Ziolkowski and A. D. Kipple, "Application of Double Negative Materials to Increase the Power Radiated by Electrically Small Antennas," IEEE Transactions on Antennas and Propagation, Vol. 51, No. 10, pp. 2626-2640, October 2003.

[4] N. Engheta, "An Idea for Thin Subwavelength Cavity Resonators Using Metamaterials With Negative Permittivity and Permeability," IEEE Antennas and Wireless Propagation Letters, Vol. 1, No. 1, pp. 10-13, 2002. 